\title{
Severe Genotype, Pancreatic Insufficiency and Low Dose of Pancreatic Enzymes Associate with Abnormal Serum Sterol Profile in Cystic Fibrosis
}

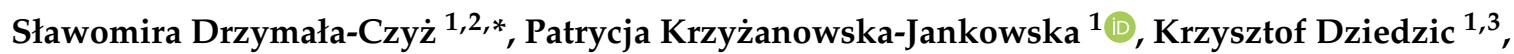 \\ Aleksandra Lisowska ${ }^{1}$, Szymon Kurek ${ }^{1}$, Joanna Goździk-Spychalska ${ }^{4}{ }^{\oplus}$, Victoria Kononets ${ }^{5}$, \\ Dagmara Woźniak ${ }^{2}$, Edyta Mądry ${ }^{6}$ and Jarosław Walkowiak ${ }^{1}$ (D)
}

check for

updates

Citation: Drzymała-Czyż, S.; Krzyżanowska-Jankowska, P.;

Dziedzic, K.; Lisowska, A.; Kurek, S.; Goździk-Spychalska, J.; Kononets, V.; Woźniak, D.; Mądry, E.; Walkowiak, J. Severe Genotype, Pancreatic Insufficiency and Low Dose of Pancreatic Enzymes Associate with Abnormal Serum Sterol Profile in Cystic Fibrosis. Biomolecules 2021, 11, 313. https://doi.org/10.3390/ biom 11020313

Received: 5 January 2021

Accepted: 16 February 2021

Published: 19 February 2021

Publisher's Note: MDPI stays neutral with regard to jurisdictional claims in published maps and institutional affiliations.

Copyright: (c) 2021 by the authors. Licensee MDPI, Basel, Switzerland. This article is an open access article distributed under the terms and conditions of the Creative Commons Attribution (CC BY) license (https:// creativecommons.org/licenses/by/ $4.0 /)$.
1 Department of Pediatric Gastroenterology and Metabolic Diseases, Poznan University of Medical Sciences, 60-572 Poznań, Poland; pkrzyzanowska@ump.edu.pl (P.K.-J.); dziedzic@up.poznan.pl (K.D.); alisowska@ump.edu.pl (A.L.); skurek@ump.edu.pl (S.K.); jarwalk@ump.edu.pl (J.W.)

2 Department of Bromatology, Poznan University of Medical Sciences, 60-354 Poznań, Poland; dagmara.wozniak94@gmail.com

3 Department of Food Science and Nutrition, Institute of Food Technology of Plant Origin, Poznań University of Life Sciences, 60-637 Poznań, Poland

4 Department of Pulmonology, Allergology and Respiratory Oncology, Poznan University of Medical Sciences, 60-569 Poznań, Poland; jogoz@ump.edu.pl

5 Department of Natural Sciences Disciplines, West Kazakhstan Marat Ospanov Medical University, Aktobe 030012, Kazakhstan; micropaleontolog@yandex.kz

6 Department of Physiology, Poznan University of Medical Sciences, 61-781 Poznań, Poland; emadry@ump.edu.pl

* Correspondence: drzymala@ump.edu.pl; Tel.: +48-608-108-427

Abstract: Background: Several factors could lead to lipid disturbances observed in cystic fibrosis (CF). This study aimed to assess sterol homeostasis in CF and define potential exogenous and endogenous determinants of lipid dysregulation. Methods: The study involved 55 CF patients and 45 healthy subjects (HS). Sterol concentrations ( $\mu \mathrm{g} / \mathrm{dL}$ ) were measured by gas chromatography/mass spectrometry. CF was characterised by lung function, pancreatic status, liver disease and diabetes coexistence, Pseudomonas aeruginosa colonisation and BMI. CFTR genotypes were classified as severe or other. Results: Campesterol and $\beta$-sitosterol concentrations were lower $(p=0.0028$ and $p<0.0001$, respectively) and lathosterol levels (reflecting endogenous cholesterol biosynthesis) were higher ( $p=$ 0.0016) in CF patients than in HS. Campesterol and $\beta$-sitosterol concentrations were lower in patients with a severe CFTR genotype, pancreatic insufficiency and lower pancreatic enzyme dose (lipase units/gram of fat). In multiple regression analyses, $\beta$-sitosterol and campesterol concentrations were predicted by genotype and pancreatic insufficiency, whereas cholesterol and its fractions were predicted by phytosterol concentrations, age, dose of pancreatic enzymes, nutritional status and genotype. Conclusions: Independent determinants of lipid status suggest that malabsorption and pancreatic enzyme supplementation play a significant role in sterol abnormalities. The measurement of campesterol and $\beta$-sitosterol concentrations in CF patients may serve for the assessment of the effectiveness of pancreatic enzyme replacement therapy and/or compliance, but further research is required.

Keywords: campesterol; $\beta$-sitosterol; stigmasterol; cholesterol; lathosterol

\section{Introduction}

Cystic fibrosis (CF) is the most common genetic disease inherited in an autosomal recessive manner caused by mutations in the CFTR (CF transmembrane conductance regulator) gene located on the long arm of the seventh chromosome. The product of this gene is the CFTR protein present on the apical surface of the epithelial cells of the respiratory 
system, pancreas, intestine and sweat glands, where it acts as a chloride $(\mathrm{Cl})$ channel [1,2]. The loss of functional CFTR $\mathrm{Cl}^{-}$channels disrupts $\mathrm{Cl}^{-}$transport across epithelia, which contributes to the production of thick, sticky mucus in secretory organs. CF involves the coexistence of pathological changes in various systems, including the liver and bile ducts [3-5], which directly affect fat metabolism. Lipid disorders in patients with CF have become a current topic of research; however, the causes of CF-related hypocholesterolemia remain unknown [6-8]. Studies conducted in animals and in vivo models with CF cells indicate that CFTR mutations affect the displacement of cholesterol to the cell membrane; in turn, cholesterol exhaustion leads to increased de novo cholesterol synthesis to restore the membrane content $[9,10]$.

The observed lipid disturbances can be explained, at least in part, by the high fat intake recommended in CF and coexisting cholestasis [11,12]. Among the most important sources of fat in the CF diet are plant oils rich in phytosterols (such as campesterol, $\beta$ sitosterol, stigmasterol) [13], which regulate the circulation of cholesterol and lower its concentration in the blood. Plant sterols inhibit the absorption of cholesterol and can displace cholesterol from micelles because they are more hydrophobic than cholesterol and have a higher affinity for these micelles. Decreased cholesterol absorption results in increased endogenous biosynthesis. Physiologically, the excess of cholesterol produced is excreted with the bile and then reabsorbed into the bloodstream [14,15]. However, high phytosterol consumption can hinder reverse absorption and, at the same time, increase endogenous cholesterol production [16,17]. Simultaneous determination of plant sterols ${ }^{\prime}$ concentrations and the estimation of their consumption in patients with CF may help to understand the impact of nutrition on the observed fat disturbances.

Only two studies conducted by Gelzo et al. have assessed the total sterol profile in CF patients. In the first small study $(n=26)$, the authors suggested that sterol absorption is reduced and synthesis is increased in CF [18]. In the second study, the sterol profile was analysed in CF patients with pancreatic sufficiency and insufficiency [19], with lower cholesterol and phytosterol levels observed in pancreatic-insufficient patients. However, nutrition and enzyme therapy were not considered. Moreover, multiple analyses were not performed, which disenables the reliable assessment of lipid status in CF.

Our study is the first to define the exogenous and endogenous determinants of sterol abnormalities in CF patients using multiple regression analysis, simultaneously taking into account dietary sterol intake and pancreatic enzyme replacement therapy.

\section{Material and Methods}

\subsection{Patients}

The study comprised 55 CF patients ( 33 female, 22 male) aged 16 to 50 years. The inclusion criteria for $\mathrm{CF}$ patients were $\mathrm{CF}$ diagnosis compatible with the $\mathrm{CF}$ Foundation guidelines [20] and age $\geq 16$ years. The exclusion criteria included pregnancy, non-CF gastrointestinal diseases with maldigestion and malabsorption, liver transplantation, other severe systemic diseases (neoplasms, endocrinopathies, liver cirrhosis) and lung or/and liver transplantation. The control group included 45 young adults ( 26 female, 19 male) aged 18 to 34 years. HS were excluded if they had a systemic/chronic/severe disease or a family history of hypercholesterolemia, or if they followed a restricted diet (e.g., any type of vegetarian diet).

The body weight and height of all participants were measured, and BMI (body mass index, $\mathrm{kg} / \mathrm{m}^{2}$ ) was calculated. Forty-eight CF patients were pancreatic-insufficient (faecal elastase- 1 concentrations $<100 \mu \mathrm{g} / \mathrm{g}$ ) [21,22] and took pancreatic enzymes. Individual CF characteristics were assessed (liver disease, diabetes, chronic and/or intermittent colonisation by Pseudomonas aeruginosa, forced expiratory volume in one second (FEV1) $[23,24]$ ) as well as genotypes, which were as follows: F508del/F508del $(n=17)$, F508del $/ 3849+10 \mathrm{kbC}$ $>\mathrm{T}(n=6)$, F508del/W1282x ( $n=2)$, F508del/CFTRdel2,3(21kb) $(n=3)$, F508del/2143delT $(n=1)$, F508del $/ 3600+2$ insT $(n=1)$, F508del/R352Q $(n=1)$, F508del/G551D $(n=1)$, F508del/N1303K $(n=1)$, F508del/3121-2A > G $(n=1)$, F508del/2183AA-G $(n=3)$, 
F508del/R851X $(n=1)$, F508del/1717-1G- > A $(n=1)$, F508del/- $(n=5), 3849+10 \mathrm{kbC}>$ $\mathrm{T} / 3849+10 \mathrm{kbC}>\mathrm{T}(n=2), 3849+10 \mathrm{kbC}>\mathrm{T} /$ dele $2,3(21 \mathrm{~kb})(n=1), 3849+10 \mathrm{kbC}>\mathrm{T} / 3600$ $+1 \mathrm{G}>\mathrm{T}(n=1), 3849+10 \mathrm{kbC}>\mathrm{T} /-(n=1), \mathrm{N} 1303 \mathrm{~K} / \mathrm{CFTRdele} 2,3(21 \mathrm{~kb})(n=1), 1524$ $+1 \mathrm{G}>\mathrm{A} / 3944$ delGT;406-6T $>\mathrm{C}(n=1), 3272-26 \mathrm{~A}>\mathrm{A} /-(n=1)$, none detected - $/-(n=$ 3) $[25,26]$. The genotypes were classified by known clinical impact: severe/severe mutations (two class I-III mutations) and other mutations (at least one class IV-VI or unknown mutation) $[27,28]$.

\subsection{Sterols}

Serum sterols were assessed using the method described previously by Corso et al. [29]. Blood samples were collected after overnight fasting, then $1 \mathrm{~N} \mathrm{KOH}$ in $90 \%$ ethanol $(3 \mathrm{~mL})$ was added to $50 \mu \mathrm{L}$ serum spiked with $100 \mu \mathrm{L}$ of an internal standard ( $5 \alpha$-cholestane). Samples were hydrolysed for $60 \mathrm{~min}$ at $80{ }^{\circ} \mathrm{C}$, then diluted with $3 \mathrm{~mL}$ of distilled water. Next, the samples were extracted three times using $2 \mathrm{~mL}$ of hexane. The upper phases were evaporated using nitrogen, then mixed in $100 \mu \mathrm{L}$ of BSTFA and pyridine (7:3; v/v), before they were dried under nitrogen and redissolved in dichloromethane $(100 \mu \mathrm{L})$. Samples $(1 \mu \mathrm{L})$ were subjected to gas chromatography with mass spectrometry (GC-MS, Agilent 7890 series II and 5975C, Agilent Technologies, Santa Clara, USA) using the HP-5ms column (Agilent J\&W GC Columns, Folsom, CA, USA). Peak integration was performed using MSD ChemStation (Agilent Technologies, Santa Clara, USA) and the concentrations of campesterol, $\beta$-sitosterol, stigmasterol and lathosterol were determined. The synthesis/absorption ratios (lathosterol/campesterol and lathosterol/ $\beta$-sitosterol) were calculated to evaluate cholesterol metabolism.

\subsection{Lipid Profile}

Total serum cholesterol was measured by an enzymatic method employing cholesterol esterase, cholesterol oxidase and the Trinder reaction using the Integrated Chemistry System Analyser (Siemens Healthcare $\mathrm{GmbH}$, Erlangen, Germany). Low-density lipoprotein cholesterol (LDL-C) and high-density lipoprotein cholesterol (HDL-C) concentrations were assessed directly using the catalase elimination method.

\subsection{Dietary Intake}

The average daily intake of energy and lipids was calculated in 45 CF patients and 35 HS. The diet was analysed based on nutritional records collected via a 3-day diary ( 2 working days and one during the weekend; Dietetyk 2015 software; Jumar, Poznań, Poland). In CF patients, a standard dose of enzyme supplementation was calculated per gram of fat consumed. Regarding phytosterols, our own database was used based on a review of national and world literature of the content of phytosterols in food products of plant origin $[30,31]$. The NutritionData.com database was also applied in the study.

\subsection{Statistical Methods}

The sterol concentrations are expressed as $\mu \mathrm{g} / \mathrm{dL}$ of serum and the concentrations of TG, total cholesterol and its fractions are presented as $\mathrm{mg} / \mathrm{dL}$. For all parameters, medians and $1^{\text {st }}-3^{\text {rd }}$ quartiles were calculated unless indicated otherwise. The Shapiro-Wilk test was used to check the normality of the data distribution, with the Mann-Whitney U-test or Fisher's exact test used to assess differences between groups. The linear correlation between sterols intake and serum sterol concentrations (lathosterol and phytosterol/cholesterol and their fractions) was analysed using Spearman's test. The relationship between the phytosterols, lathosterol and cholesterol and its fractions and all studied clinical parameters and nutritional intake was assessed using a multiple linear logistic regression (stepwise and backward). The following variables were interpreted as independent in all regression models: age, sex, BMI, CFTR genotype (severe/severe vs. other), $\mathrm{FEV}_{1}$, pancreatic insufficiency, dose of pancreatic enzymes (lipase units/gram of fat), liver disease, diabetes, $P$. aeruginosa colonisation. For cholesterol and its fractions, sterol concentrations were 
also included. A $p$-value $<0.05$ was considered statistically significant. In the graphic presentation, the relationship between the pancreatic enzyme dose and total cholesterol concentrations was smoothed using the LOWESS method. All statistical analyses were conducted using GraphPad Prism 5.01 (GraphPad Software, Inc., La Jolla, CA, USA) and Statistica 12.0 software (StatSoft Inc., Tulsa, USA).

\subsection{Ethical Considerations}

All participants gave their informed written consent for participation in the study. The study was approved by the Bioethical Committee of the Poznan University of Medical Sciences, Poznań, Poland (decision no 1225/16). The project was conducted in accordance with the Declaration of Helsinki.

\section{Results}

The anthropometric and clinical characteristics of the study groups are presented in Table 1 . The CF patients had a significantly lower median of body height, weight and BMI than the HS group ( $p=0.0022, p=0.0295, p=0.026$, respectively).

Table 1. Anthropometric and clinical data of cystic fibrosis (CF) patients and healthy subjects (HS).

\begin{tabular}{cccc}
\hline Parameters & CF Patients & HS & $P$ \\
\hline N & \multicolumn{2}{c}{ Median $(1$ st-3rd quartile) } & \\
\hline Age [years] & 55 & 45 & - \\
& 26.9 & 25.4 & ns. \\
Sex ratio [F/M] & $(20.5-32.0)$ & $(22.4-28.3)$ & ns. \\
Body height [m] & $33 / 22$ & $26 / 19$ & \\
& $(60.0 \%)$ & $(57.8 \%)$ & 0.0295 \\
Body weight [kg] & 1.69 & $(1.6-1.8)$ & 0.0022 \\
& $(1.6-1.7)$ & 65.0 & \\
BMI [kg/m $\left.{ }^{2}\right]$ & 57.0 & $(57.2-75.5)$ & 0.0260 \\
Pancreatic insufficiency & $(51.4-65.0)$ & 21.9 & - \\
FEV1 [\%] & 20.5 & - & - \\
\hline
\end{tabular}

BMI—body mass index. FEV1—forced expiratory volume in one second.

Table 2 presents a comparison of all measured sterol concentrations between CF patients and HS. The concentrations of campesterol and $\beta$-sitosterol were lower in the CF patients ( $p=0.0028$ and $p<0.0001$, respectively). The concentration of stigmasterol was comparable between analysed groups, whereas the level of lathosterol (a marker of endogenous cholesterol biosynthesis) was significantly higher in CF patients $(p=0.0016)$. Other sterols measured by the enzymatic methods were lower in CF patients (for total cholesterol $p=0.006$, for HDL-C $p<0.0001$, for LDL-C $p=0.0093$ ). The synthesis/absorption ratios of sterols (lathosterol/campesterol and lathosterol/ $\beta$-sitosterol) were higher in CF patients than HS ( $p=0.0002$ and $p<0.0001$, respectively). The box plot of these ratios in CF patients and HS is shown in Figure 1. 
Table 2. Sterols' concentrations in cystic fibrosis (CF) patients and healthy subjects (HS).

\begin{tabular}{|c|c|c|c|c|}
\hline \multirow{2}{*}{ Method } & Clinical Parameters & CF Patients & HS & $p$ \\
\hline & \multicolumn{4}{|c|}{ Median (1st-3rd Quartile) } \\
\hline \multirow{4}{*}{ Chromatographic } & Campesterol $[\mu \mathrm{g} / \mathrm{dL}]$ & $\begin{array}{c}292 \\
(172-492)\end{array}$ & $\begin{array}{c}452 \\
(363-514)\end{array}$ & 0.0028 \\
\hline & $\beta$-sitosterol $[\mu \mathrm{g} / \mathrm{dL}]$ & $\begin{array}{c}165 \\
(92-283)\end{array}$ & $\begin{array}{c}401 \\
(319-507)\end{array}$ & $<0.0001$ \\
\hline & Stigmasterol $[\mu \mathrm{g} / \mathrm{dL}]$ & $\begin{array}{c}9 \\
(7-24)\end{array}$ & $\begin{array}{c}14 \\
(12-19)\end{array}$ & ns. \\
\hline & Lathosterol $[\mu \mathrm{g} / \mathrm{dL}]$ & $\begin{array}{c}480 \\
(281-720)\end{array}$ & $\begin{array}{c}286 \\
(235-435)\end{array}$ & 0.0016 \\
\hline \multirow{3}{*}{ Enzymatic } & Total cholesterol [mg/dL] & $\begin{array}{c}135 \\
(110-176)\end{array}$ & $\begin{array}{c}168 \\
(147-185)\end{array}$ & 0.0006 \\
\hline & HDL-C [mg/dL] & $\begin{array}{c}49 \\
(42-56)\end{array}$ & $\begin{array}{c}66 \\
(53-67)\end{array}$ & $<0.0001$ \\
\hline & LDL-C [mg/dL] & $\begin{array}{c}66 \\
(51-94)\end{array}$ & $\begin{array}{c}83 \\
(75-105)\end{array}$ & 0.0093 \\
\hline \multirow{2}{*}{ Ratio } & Lathosterol/Campesterol & $\begin{array}{c}1.73 \\
(0.64-3.43)\end{array}$ & $\begin{array}{c}0.80 \\
(0.48-0.99)\end{array}$ & 0.0002 \\
\hline & Lathosterol/ $\beta$-sitosterol & $\begin{array}{c}2.93 \\
(1.18-6.62)\end{array}$ & $\begin{array}{c}0.91 \\
(0.54-1.15)\end{array}$ & $<0.0001$ \\
\hline
\end{tabular}

HDL-C—high-density lipoprotein cholesterol. LDL-C—low-density lipoprotein cholesterol.

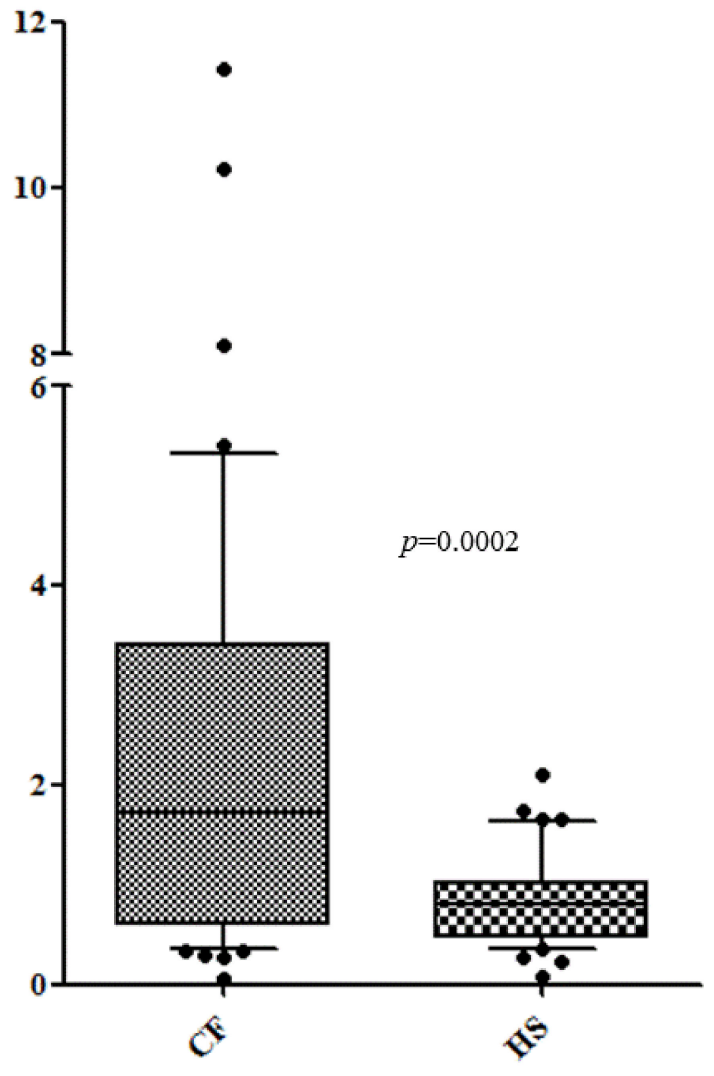

Lathosterol/campesterol ratio

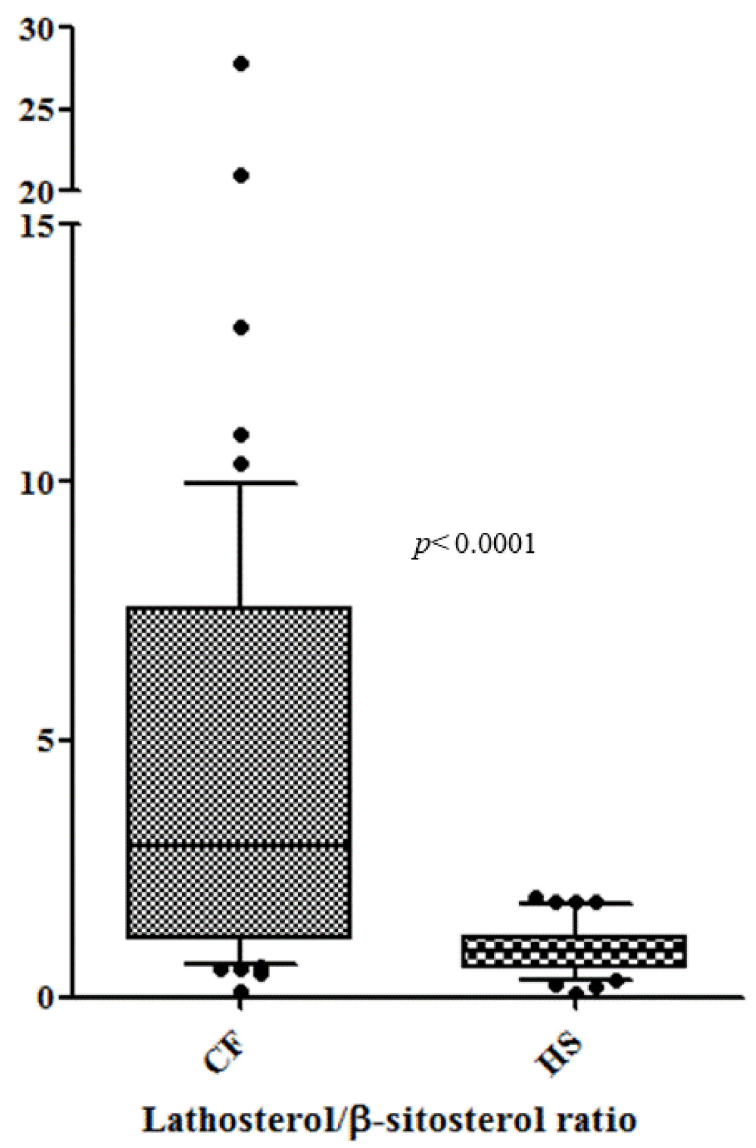

Figure 1. Lathosterol/campesterol and lathosterol/ $\beta$-sitosterol ratios in cystic fibrosis (CF) patients and healthy subjects (HS). Box and whiskers: $10^{\text {th }}-90^{\text {th }}$ percentiles and $1^{\text {st }}-3^{\text {rd }}$ quartiles are shown. 
The daily intake of energy (kcal), estimated energy requirement (\%), total fat $(\mathrm{g})$ and cholesterol (mg) intake were higher in CF patients than in HS $(p=0.0005, p=0.0004, p=$ $0.0273, p=0.0132$, respectively). In $22(40.0 \%)$ patients, the energy consumption was lower than the $110 \%$ of the RDA (Recommended Dietary Allowance), which is proposed as the minimum recommended intake for CF (independent of age, malnutrition or concurrent disease) [13]. Table 3 presents a comparison of all measured dietary intakes between CF patients and HS.

Table 3. Dietary intakes in cystic fibrosis (CF) patients and healthy subjects (HS).

\begin{tabular}{|c|c|c|c|}
\hline Parameters & CF Patients & HS & $p$ \\
\hline \multicolumn{4}{|c|}{ Median (1st-3rd Quartile) } \\
\hline $\mathrm{N}$ & 45 & 35 & - \\
\hline Energy [kcal/d] & $\begin{array}{c}2433 \\
(2114-2699)\end{array}$ & $\begin{array}{c}1850 \\
(1730-2245)\end{array}$ & 0.0005 \\
\hline EER [\%] & $\begin{array}{c}121.5 \\
(96.8-130.5)\end{array}$ & $\begin{array}{c}94.4 \\
(86.6-101.2)\end{array}$ & 0.0004 \\
\hline Total fat $[\%$ en] & $\begin{array}{c}37.3 \\
(33.5-40.5)\end{array}$ & $\begin{array}{c}39.1 \\
(33.9-45.0)\end{array}$ & ns. \\
\hline Total fat $[\mathrm{g} / \mathrm{d}]$ & $\begin{array}{c}102.2 \\
(86.4-119.5)\end{array}$ & $\begin{array}{c}84.3 \\
(71.3-107.5)\end{array}$ & 0.0273 \\
\hline Cholesterol [mg/d] & $\begin{array}{c}332.4 \\
(244.6-435.0)\end{array}$ & $\begin{array}{c}288.2 \\
(149.1-315.3)\end{array}$ & 0.0132 \\
\hline Total phytosterol $[\mathrm{mg} / \mathrm{d}]$ & $\begin{array}{c}125.2 \\
(101.5-198.8)\end{array}$ & $\begin{array}{c}151.3 \\
(131.1-214.8)\end{array}$ & ns. \\
\hline Campesterol $[\mathrm{mg} / \mathrm{d}]$ & $\begin{array}{c}28.5 \\
(22.6-44.6)\end{array}$ & $\begin{array}{c}33.7 \\
(25.6-48.8)\end{array}$ & ns. \\
\hline B-sitosterol [mg/d] & $\begin{array}{c}72.2 \\
(57.1-112.5)\end{array}$ & $\begin{array}{c}88.8 \\
(70.9-145.1)\end{array}$ & ns. \\
\hline Stigmasterol [mg/d] & $\begin{array}{c}3.1 \\
(1.8-4.5)\end{array}$ & $\begin{array}{c}4.0 \\
(3.0-6.9)\end{array}$ & ns. \\
\hline $\begin{array}{l}\text { Lipase dose } \\
\text { [U/g of fat }]\end{array}$ & $\begin{array}{c}2060 \\
(1540-3042)\end{array}$ & - & - \\
\hline
\end{tabular}

EER—estimated energy requirement. *—-for pancreatic-insufficient patients.

The correlation between total phytosterols intake and serum cholesterol concentration for CF patients was statistically significant ( $p=0.0007, \mathrm{r}=-0.4888$; Figure 2).

The sterol concentrations in subgroups of CF patients defined by the different clinical parameters are presented in Table 4 . Total cholesterol and lathosterol concentrations were lower in males. Lower campesterol, $\beta$-sitosterol, total cholesterol and LDL-C levels were documented in patients with a severe CFTR genotype and exocrine pancreatic insufficiency. Interestingly, the HDL-C concentration was lower only in patients with a severe CFTR genotype. Meanwhile, $\beta$-sitosterol concentrations were lower in patients colonised with $P$. aeruginosa. A lower lathosterol concentration was noted also in CF patients with poor lung function. 


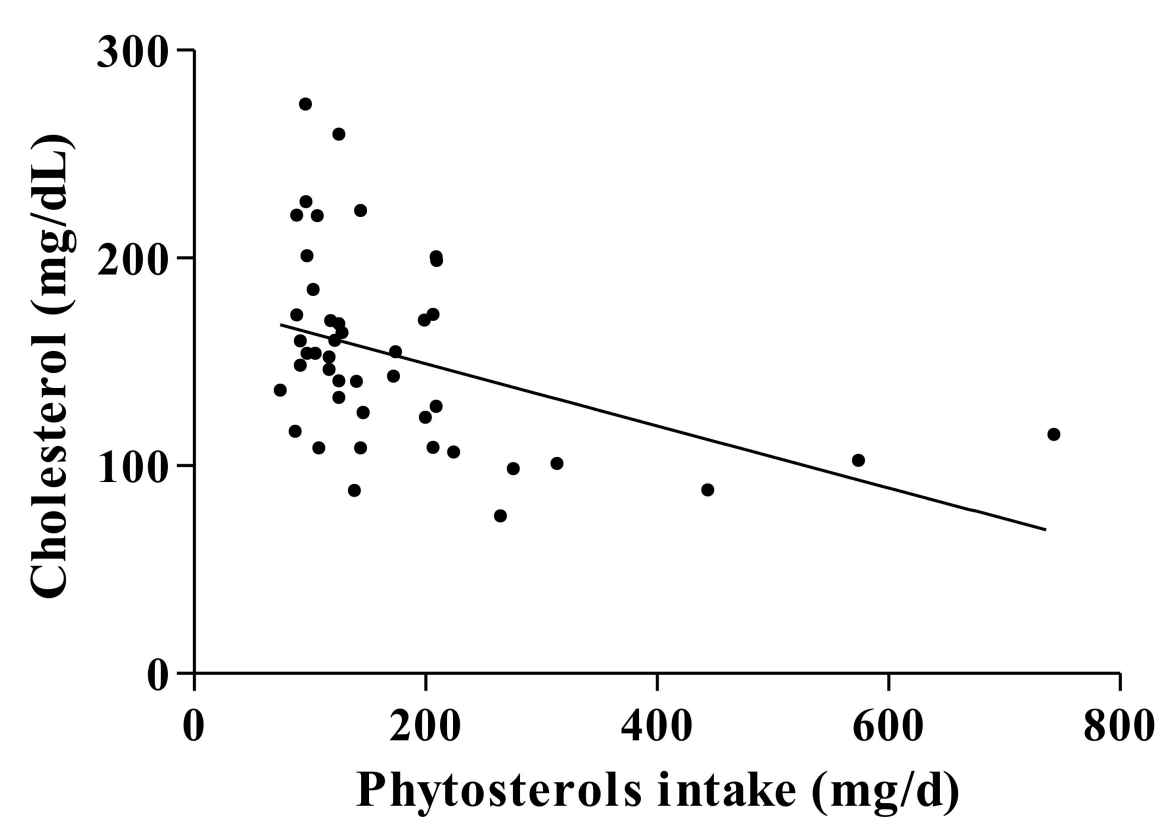

Figure 2. The relationship between phytosterols intake $(\mathrm{mg} / \mathrm{d})$ and serum cholesterol concentration $(\mathrm{mg} / \mathrm{dL})(p=0.0007, \mathrm{r}=-0.4888)$ in cystic fibrosis patients.

Forward stepwise regression revealed the following independent correlates of total cholesterol in CF: campesterol, age, sex, dose of pancreatic enzymes (lipase units/g of fat) and BMI; for HDL: age and sitosterol. Backward stepwise regression showed that the CFTR genotype and sitosterol were independent predictors of LDL concentrations (Table 5). The model seemed to explain a moderate fraction of the variability in the studied parameters.

Only one independent correlate of $\beta$-sitosterol $\left(R^{2}=0.2526, p=0.002\right)$ : CFTR genotype $(\beta=-166.75, p=0.00055)$, and campesterol $\left(R^{2}=0.2585, p=0.0017\right)$ : CFTR genotype $(\beta=$ $-192.17, p=0.035)$, was identified.

The detailed analysis of pancreatic-insufficient patients revealed significant correlations between pancreatic enzyme dose (lipase units/g of fat) and serum cholesterol concentration and its fractions $(p<0.0001, \mathrm{r}=0.5648$ for total cholesterol, $p=0.0079, \mathrm{r}=$ 0.3870 for HDL-C and $p=0.0013, \mathrm{r}=0.4596$ for LDL-C; Figure 3).

Similar associations were also documented between pancreatic enzyme dose (lipase units / $\mathrm{g}$ of fat) and phytosterol concentrations ( $p<0.0088, \mathrm{r}=0.3819$ for campesterol and $p$ $<0.0312, \mathrm{r}=0.3180$ for $\beta$-sitosterol), as presented in Figure 4 .

Subsequent analysis conducted in pancreatic-insufficient CF patients showed that the supplementation dose of $2500 \mathrm{U}$ of lipase/g of fat was significant for hypocholesterolemia (Figure 5). The differences in sterol concentrations in subgroups receiving higher and lower doses are presented in Table 6. 
Table 4. Sterol concentrations in subgroups of cystic fibrosis patients defined by different clinical parameters.

\begin{tabular}{|c|c|c|c|c|c|c|c|c|c|c|c|c|c|c|c|c|}
\hline \multirow{2}{*}{$\begin{array}{c}\text { Parameters } \\
\begin{array}{c}\text { Median } \\
1^{\text {st_-3 }}{ }^{\text {rd }} \\
\text { Quartiles }\end{array}\end{array}$} & \multicolumn{2}{|c|}{ Sex } & \multicolumn{2}{|c|}{ BMI $\left[\mathrm{kg} / \mathrm{m}^{2}\right]$} & \multicolumn{2}{|c|}{ CFTR Genotype } & \multicolumn{2}{|c|}{$\begin{array}{c}\text { Exocrine } \\
\text { Pancreatic } \\
\text { Insufficiency }\end{array}$} & \multicolumn{2}{|c|}{ Liver Disease } & \multicolumn{2}{|c|}{ Diabetes } & \multicolumn{2}{|c|}{ P. aeruginosa } & \multicolumn{2}{|c|}{ FEV1 [\%] } \\
\hline & Women & Men & $\leq 21$ & $>21$ & Severe & Other & Yes & No & Yes & No & Yes & No & Yes & No & $<80$ & $>80$ \\
\hline $\mathrm{N}$ & 33 & 22 & 28 & 27 & 32 & 23 & 48 & 7 & 26 & 29 & 9 & 46 & 37 & 18 & 39 & 16 \\
\hline $\begin{array}{c}\text { Campesterol } \\
{[\mu \mathrm{g} / \mathrm{dL}]}\end{array}$ & $\begin{array}{l}298.9 \\
207.6- \\
505.5\end{array}$ & $\begin{array}{l}356.8 \\
152.5- \\
465.1\end{array}$ & $\begin{array}{c}274.9 \\
175.0- \\
411.1\end{array}$ & $\begin{array}{l}298.9 \\
223.9- \\
565.2\end{array}$ & $\begin{array}{c}225.6 \\
* * * \\
160.2- \\
308.3\end{array}$ & $\begin{array}{c}549.8 \\
* * * \\
311.1- \\
790.6\end{array}$ & $\begin{array}{c}256.8 * * \\
163.8- \\
422.3\end{array}$ & $\begin{array}{c}733.7 * * \\
479.9- \\
1015.0\end{array}$ & $\begin{array}{l}251.8 \\
157.6- \\
402.8\end{array}$ & $\begin{array}{l}309.5 \\
227.2- \\
599.2\end{array}$ & $\begin{array}{l}221.9 \\
166.3- \\
292.4\end{array}$ & $\begin{array}{c}307.1 \\
195.6- \\
587.2\end{array}$ & $\begin{array}{l}257.3 \\
166.3- \\
431.3\end{array}$ & $\begin{array}{l}360.7 \\
221.9- \\
587.2\end{array}$ & $\begin{array}{c}301.5 \\
199.4- \\
492.0\end{array}$ & $\begin{array}{c}257.3 \\
152.6- \\
52.0\end{array}$ \\
\hline $\begin{array}{c}\beta \text {-sitosterol } \\
{[\mu \mathrm{g} / \mathrm{dL}]}\end{array}$ & $\begin{array}{l}167.9 \\
97.3- \\
296.5\end{array}$ & $\begin{array}{l}156.2 \\
87.4- \\
283.6\end{array}$ & $\begin{array}{l}159.5 \\
86.4- \\
241.5\end{array}$ & $\begin{array}{l}20.1 .6 \\
97.3- \\
289.4\end{array}$ & $\begin{array}{c}108.3 \\
* * * \\
81.1- \\
210.2\end{array}$ & $\begin{array}{c}273.3 \\
* * * \\
172.4- \\
376.8\end{array}$ & $\begin{array}{c}156.2 * * \\
85.5- \\
246.1\end{array}$ & $\begin{array}{c}362.7 * * \\
244.7- \\
520.1\end{array}$ & $\begin{array}{l}113.6 \\
81.7- \\
221.4\end{array}$ & $\begin{array}{l}214.6 \\
114.8- \\
285.5\end{array}$ & $\begin{array}{l}160.1 \\
101.7- \\
170.3\end{array}$ & $\begin{array}{l}193.5 \\
91.8- \\
291.3\end{array}$ & $\begin{array}{c}126.1 * \\
87.4- \\
267.1\end{array}$ & $\begin{array}{c}207.4 \\
156.2- \\
283.6\end{array}$ & $\begin{array}{l}165.4 \\
95.6- \\
265.1\end{array}$ & $\begin{array}{l}159.1 \\
86.4- \\
297,3\end{array}$ \\
\hline $\begin{array}{l}\text { Stigmasterol } \\
{[\mu \mathrm{g} / \mathrm{dL}]}\end{array}$ & $\begin{array}{l}10.4 \\
7.7- \\
23.9\end{array}$ & $\begin{array}{l}9.1 \\
7.0- \\
23.7\end{array}$ & $\begin{array}{l}12.7 \\
8.5- \\
24.1\end{array}$ & $\begin{array}{l}8.2 \\
7.2- \\
23.5\end{array}$ & $\begin{array}{c}9.0 \\
7.2-23.5\end{array}$ & $\begin{array}{c}9.8 \\
7.6-23.7\end{array}$ & $\begin{array}{c}8.4 \\
7.2-23.0\end{array}$ & $\begin{array}{c}20.9 \\
9.8-27.6\end{array}$ & $\begin{array}{c}9.2 \\
7.5-20.9\end{array}$ & $\begin{array}{c}8.8 \\
7.2-23.8\end{array}$ & $\begin{array}{c}5.9 \\
5.0-6.8\end{array}$ & $\begin{array}{c}9.2 \\
7.4-23.8\end{array}$ & $\begin{array}{c}12.1 \\
8.4-24.6\end{array}$ & $\begin{array}{c}7.8 \\
6.2-9.5\end{array}$ & $\begin{array}{c}9.1 \\
7.1-23.0\end{array}$ & $\begin{array}{c}8.4 \\
7.8-24.5\end{array}$ \\
\hline $\begin{array}{c}\text { Lathosterol } \\
{[\mu \mathrm{g} / \mathrm{dL}]}\end{array}$ & $\begin{array}{l}543.3 * \\
414.8- \\
814.2\end{array}$ & $\begin{array}{l}381.7 \\
253.3- \\
531.5\end{array}$ & $\begin{array}{l}448.3 \\
242.5- \\
618.7\end{array}$ & $\begin{array}{l}569.3 \\
388.9- \\
790.1\end{array}$ & $\begin{array}{l}489.7 \\
263.0- \\
735.4\end{array}$ & $\begin{array}{l}476.5 \\
260.9- \\
704.8\end{array}$ & $\begin{array}{l}486.5 \\
300.1- \\
739.4\end{array}$ & $\begin{array}{l}472.8 \\
287.7- \\
506.1\end{array}$ & $\begin{array}{l}505.8 \\
292.9- \\
722.7\end{array}$ & $\begin{array}{l}480.1 \\
317.1- \\
717.5\end{array}$ & $\begin{array}{c}314.6 \\
199.1- \\
468.5\end{array}$ & $\begin{array}{l}493.0 \\
372.1- \\
731.5\end{array}$ & $\begin{array}{l}480.2 \\
257.0- \\
696.4\end{array}$ & $\begin{array}{l}493.0 \\
372.1- \\
819.1\end{array}$ & $\begin{array}{c}432.9 * \\
258.8- \\
604.4\end{array}$ & $\begin{array}{l}696.4 * \\
510.1- \\
814.3\end{array}$ \\
\hline $\begin{array}{c}\text { Total } \\
\text { cholesterol } \\
{[\mathrm{mg} / \mathrm{dL}]}\end{array}$ & $\begin{array}{l}143.0 * \\
131.0- \\
182.0\end{array}$ & $\begin{array}{c}123.0 * \\
91.0- \\
166.0\end{array}$ & $\begin{array}{l}135.0 \\
97.2- \\
152.0\end{array}$ & $\begin{array}{l}157.0 \\
123.2- \\
186.0\end{array}$ & $\begin{array}{l}125.0 \\
* * * \\
94.0- \\
136.0\end{array}$ & $\begin{array}{c}184.0 \\
* * * \\
144.5- \\
195.5\end{array}$ & $\begin{array}{l}135.0 * \\
103.5- \\
167.5\end{array}$ & $\begin{array}{c}182.0 * \\
146.5- \\
205.5\end{array}$ & $\begin{array}{l}135.0 \\
98.7- \\
158.2\end{array}$ & $\begin{array}{l}144.0 \\
125.5- \\
186.0\end{array}$ & $\begin{array}{l}123.0 \\
98.0- \\
161.0\end{array}$ & $\begin{array}{l}135.0 \\
124.0- \\
186.0\end{array}$ & $\begin{array}{l}135.0 \\
101.0- \\
166.0\end{array}$ & $\begin{array}{c}161.0 \\
124.0- \\
186.0\end{array}$ & $\begin{array}{l}135.0 \\
105.0- \\
172.0\end{array}$ & $\begin{array}{l}135.0 \\
126.0- \\
184.0\end{array}$ \\
\hline $\begin{array}{l}\text { HDL-C } \\
{[\mathrm{mg} / \mathrm{dL}]} \\
\text { LDL-C } \\
{[\mathrm{mg} / \mathrm{dL}]}\end{array}$ & $\begin{array}{l}51.0 \\
43.0- \\
57.0 \\
71.0 \\
57.0- \\
107.0\end{array}$ & $\begin{array}{l}47.0 \\
37.8- \\
55.3 \\
57.5 \\
44.3- \\
91.3\end{array}$ & $\begin{array}{l}45.0 \\
34.8- \\
58.0 \\
61.5 \\
50.3- \\
74.3\end{array}$ & $\begin{array}{l}49.5 \\
44.0- \\
55.0 \\
90.5 \\
56.3- \\
109.8\end{array}$ & $\begin{array}{c}44.5 * * \\
33.3- \\
51.5 \\
55.0 * * * \\
42.5- \\
69.5\end{array}$ & $\begin{array}{c}54.5 * * \\
48.3- \\
57.8 \\
95.0 * * * \\
76.0- \\
111.0\end{array}$ & $\begin{array}{c}48.0 \\
41.5- \\
55.0 \\
\mathbf{6 2 . 0 * *} \\
\mathbf{4 8 . 0 -} \\
\mathbf{8 7 . 0}\end{array}$ & $\begin{array}{c}53.0 \\
50.5- \\
57.5 \\
\mathbf{1 0 9 . 0}^{* *} \\
\mathbf{9 5 . 0}^{*} \\
\mathbf{1 2 6 . 0}\end{array}$ & $\begin{array}{l}46.5 \\
35.3- \\
55.5 \\
62.0 \\
51.0- \\
89.3\end{array}$ & $\begin{array}{l}49.5 \\
43.0- \\
55.5 \\
76.0 \\
55.0- \\
109.0\end{array}$ & $\begin{array}{l}48.0 \\
37.0- \\
59.0 \\
62.0 \\
51.0- \\
83.0\end{array}$ & $\begin{array}{l}49.0 \\
42.0- \\
55.5 \\
70.0 \\
55.0- \\
104.0\end{array}$ & $\begin{array}{l}48.0 \\
36.0- \\
54.0 \\
62.0 \\
49.0- \\
91.0\end{array}$ & $\begin{array}{l}51.0 \\
47.0- \\
56.0 \\
82.0 \\
57.8- \\
109.3\end{array}$ & $\begin{array}{l}47.0 \\
36.5- \\
56.0 \\
68.0 \\
51.0- \\
93.5\end{array}$ & $\begin{array}{l}51.5 \\
47.0- \\
58.5 \\
65.5 \\
52.8- \\
95.0\end{array}$ \\
\hline
\end{tabular}

${ }^{*} p<0.05 ;{ }^{* *} p<0.01 ;{ }^{* * *} p<0.001$; HDL-C-high-density lipoprotein cholesterol; LDL-C-low-density lipoprotein cholesterol. 
Table 5. Forward (total cholesterol and HDL) and backward (LDL) stepwise regression analyses of various factors predictive of cholesterol and its fractions in cystic fibrosis patients.

\begin{tabular}{|c|c|c|c|c|c|}
\hline Dependent Variable & $p$ Model & $\mathbf{R}^{2}$ & $\begin{array}{c}\text { Independent } \\
\text { Variables }\end{array}$ & $\beta$ & $p$ \\
\hline \multirow{5}{*}{ Total cholesterol } & \multirow{5}{*}{$10^{-6}$} & \multirow{5}{*}{0.6242} & Campesterol & 0.0686 & 0.000046 \\
\hline & & & Age & 2.0042 & 0.0011 \\
\hline & & & Sex & 26.1551 & 0.0048 \\
\hline & & & $\begin{array}{c}\mathrm{U} \text { of lipase } / \mathrm{g} \\
\text { of fat }\end{array}$ & 0.0073 & 0.032 \\
\hline & & & BMI & 3.1605 & 0.040 \\
\hline \multirow{2}{*}{ HDL-C } & \multirow{2}{*}{0.0143} & \multirow{2}{*}{0.3841} & Age & 0.63768 & 0.025 \\
\hline & & & $\beta$-sitosterol & 0.04741 & 0.048 \\
\hline \multirow[t]{2}{*}{ LDL-C } & \multirow[t]{2}{*}{$10^{-6}$} & \multirow[t]{2}{*}{0.5075} & $\begin{array}{c}\text { CFTR } \\
\text { genotype }\end{array}$ & -29.5117 & 0.00011 \\
\hline & & & $\beta$-sitosterol & 0.0665 & 0.0016 \\
\hline
\end{tabular}

HDL-C—high-density lipoprotein cholesterol; LDL-C-low-density lipoprotein cholesterol.

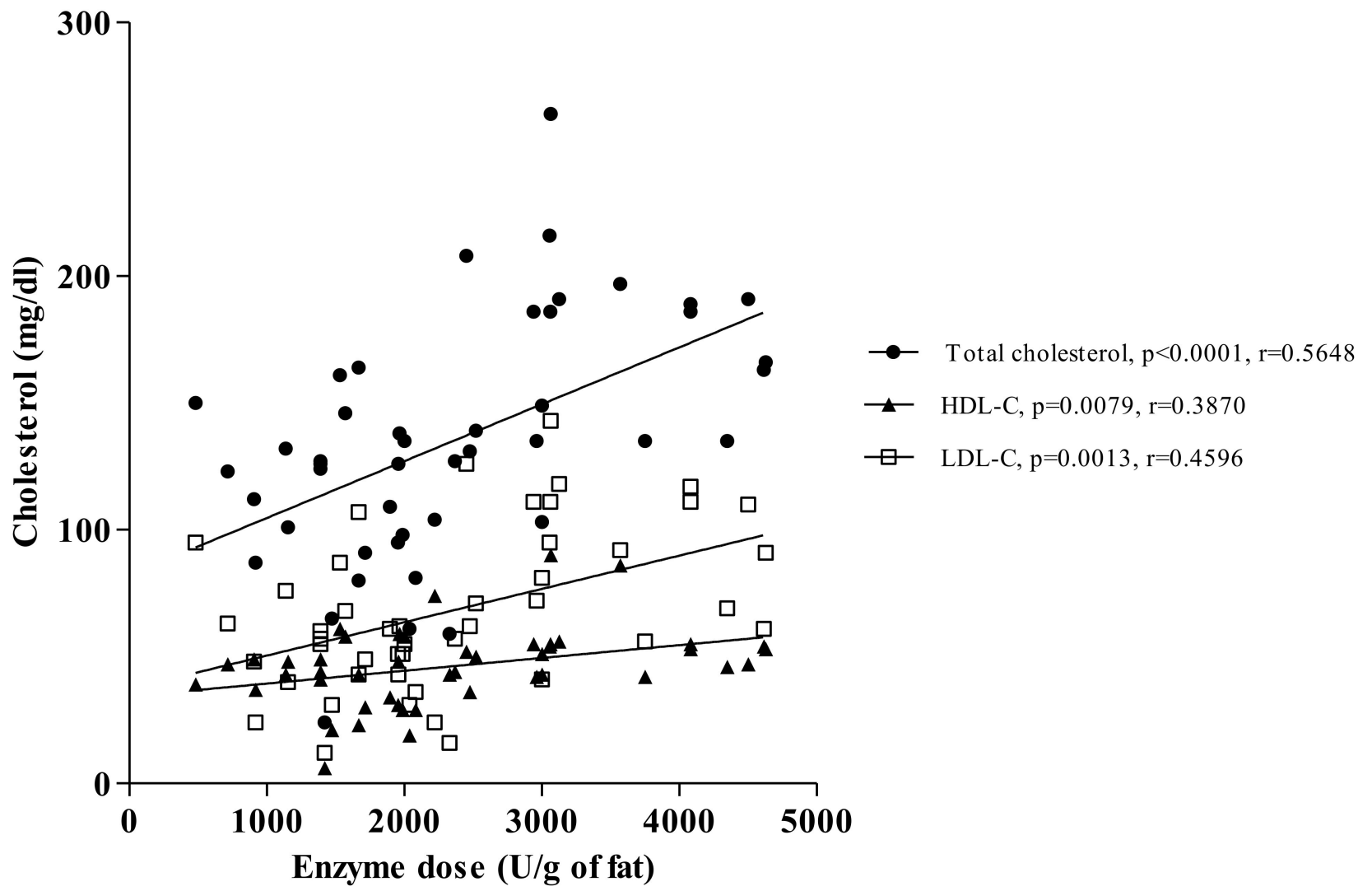

Figure 3. The relationships between enzyme intake (lipase units/g of fat) and serum cholesterol concentration (mg/dL) in pancreatic-insufficient patients. 


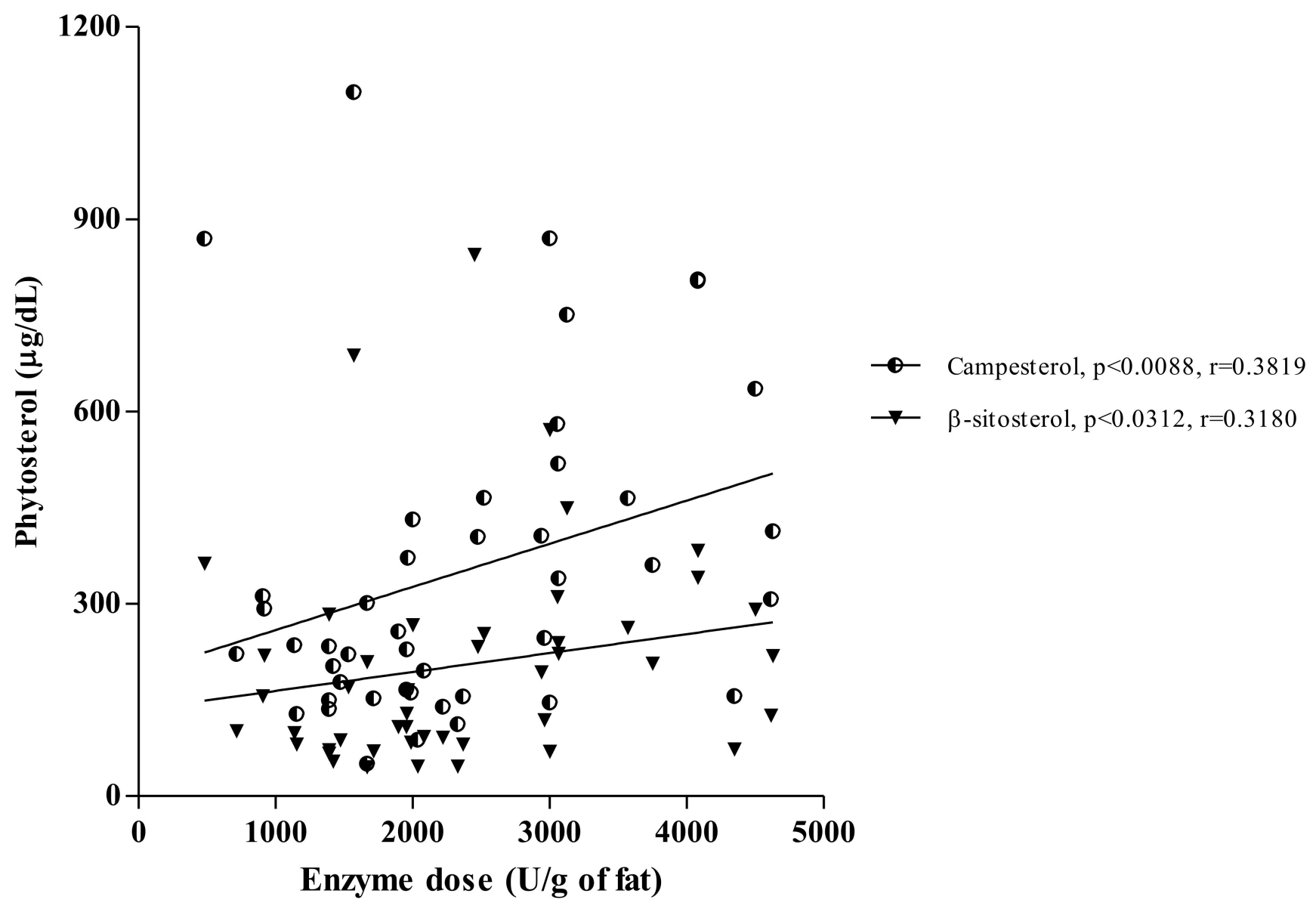

Figure 4. The relationships between enzyme intake (lipase units/g of fat) and phytosterol concentrations ( $\mu \mathrm{g} / \mathrm{dL})$ in pancreatic-insufficient patients.

Table 6. Sterol concentrations in subgroups of cystic fibrosis patients supplemented with higher and lower pancreatic enzyme doses.

\begin{tabular}{|c|c|c|c|}
\hline $\begin{array}{c}\text { Enzyme Dose } \\
\text { [Lipase units/g of Fat] }\end{array}$ & $\leq \mathbf{2 5 0 0}$ & $>2500$ & $P$ \\
\hline \multicolumn{4}{|c|}{ Median (1st-3rd quartile) } \\
\hline $\mathrm{N}$ & 28 & 17 & - \\
\hline Campesterol $[\mu \mathrm{g} / \mathrm{dL}]$ & $\begin{array}{c}221.1 \\
(152.5-301.5)\end{array}$ & $\begin{array}{c}465.1 \\
(340.0-635.4)\end{array}$ & 0.0014 \\
\hline$\beta$-sitosterol $[\mu \mathrm{g} / \mathrm{dL}]$ & $\begin{array}{c}101.7 \\
(81.0-209.7)\end{array}$ & $\begin{array}{c}238.9 \\
(193.5-311.0)\end{array}$ & 0.0078 \\
\hline Stigmasterol $[\mu \mathrm{g} / \mathrm{dL}]$ & $\begin{array}{c}9.2 \\
(8.3-23.5)\end{array}$ & $\begin{array}{c}7.5 \\
(7.1-12.1)\end{array}$ & ns. \\
\hline Total cholesterol [mg/dL] & $\begin{array}{c}123.0 \\
(91.0-132.2)\end{array}$ & $\begin{array}{c}186.1 \\
(139.2-191.0)\end{array}$ & $<0.0001$ \\
\hline Lathosterol $[\mu \mathrm{g} / \mathrm{dL}]$ & $\begin{array}{c}486.5 \\
(314.6-707.5)\end{array}$ & $\begin{array}{c}480.2 \\
(261.4-761.8)\end{array}$ & ns. \\
\hline
\end{tabular}




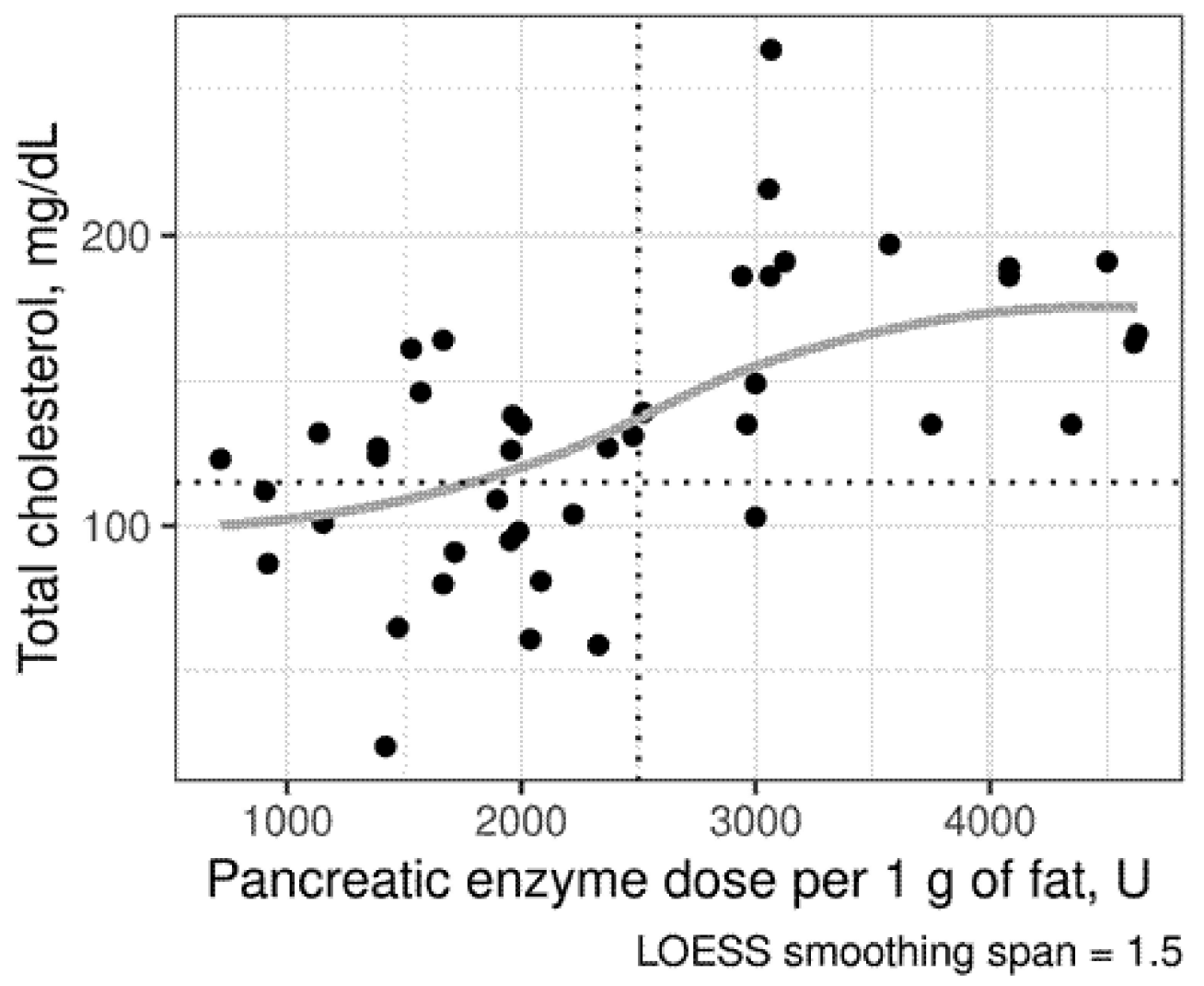

Figure 5. Scatterplot of total cholesterol in pancreatic-insufficient (PI) patients with cystic fibrosis (CF) with different doses of pancreatic enzymes. Points below the dotted horizontal line indicate patients with hypocholesterolemia as defined by the local laboratory reference range $(<115 \mathrm{mg} / \mathrm{dL}$ or $3 \mathrm{mmol} / \mathrm{L})$. The vertical line was set at the enzyme dose of 2750 lipase units/g of fat, above which only one patient experienced hypocholesterolemia. The lower rate of hypocholesterolemia in PI $\mathrm{CF}$ patients receiving $>2500 \mathrm{U} / \mathrm{d}$ of pancreatic enzyme replacement therapy was confirmed by Fisher's test (two-sided $p=$ 0.0026). Locally estimated scatterplot smoothing (LOWESS) curve is displayed in grey.

Total cholesterol and the sum of phytosterol concentrations in pancreatic-insufficient (PI) CF patients supplemented with a lower $(\leq 2500 \mathrm{U}$ of lipase/g of fat) and higher $(>2500$ $\mathrm{U}$ of lipase/g of fat) dose of pancreatic enzymes, pancreas-sufficient CF patients and HS are presented in Figure 6, showing that total cholesterol and the sum of phytosterol concentrations were significantly lower in the patients supplemented with a lower dose of the enzyme $(p<0.0001)$. 


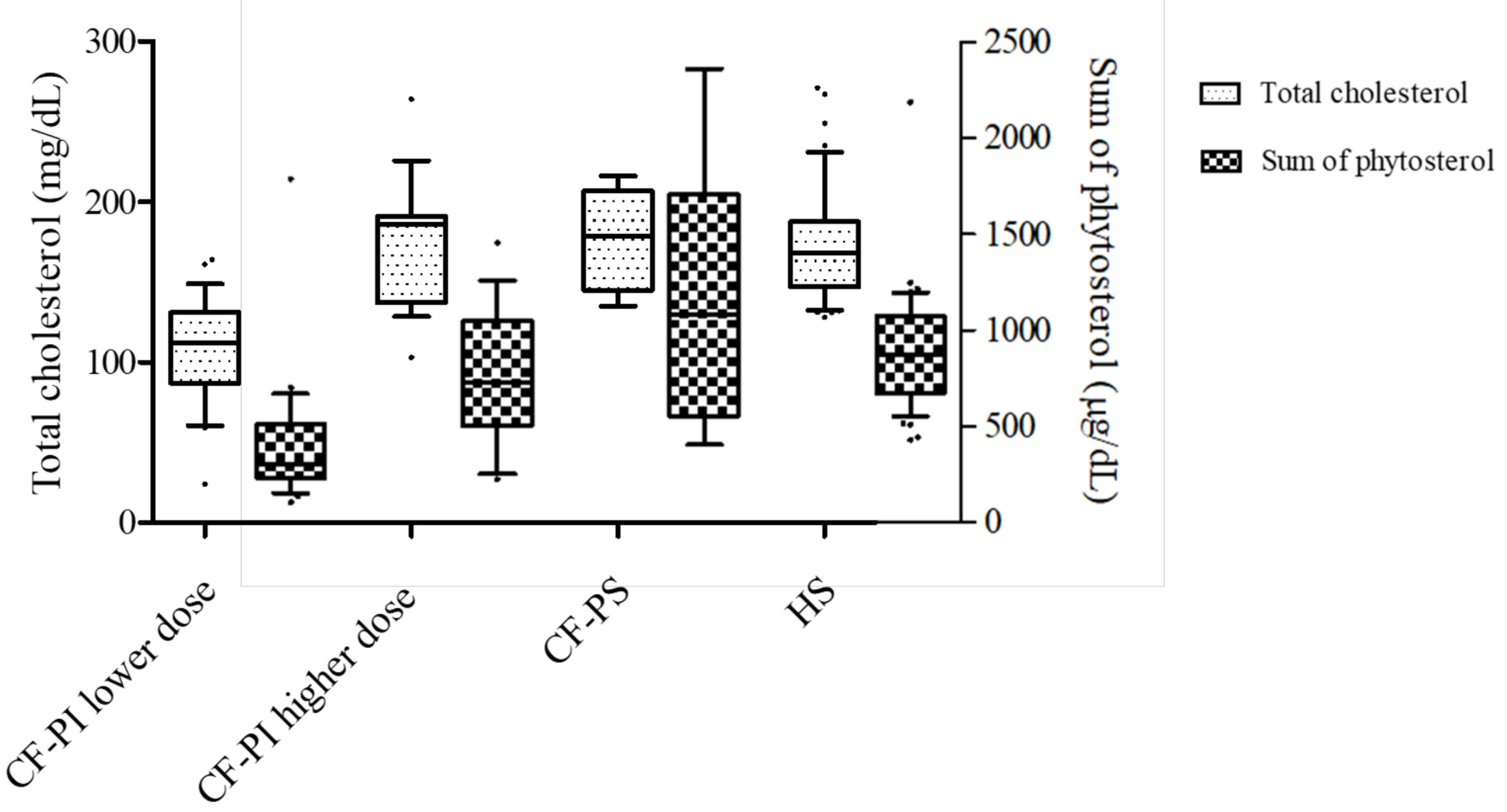

Figure 6. Sterol concentrations in cystic fibrosis patients: pancreatic-insufficient (CF-PI) subjects supplemented with lower ( $\leq 2500 \mathrm{U}$ of lipase/g of fat) and higher (>2500 U of lipase/g of fat) doses of enzymes, pancreatic-sufficient (CF-PS) subjects and healthy subjects (HS). Total cholesterol $\left(^{*}\right)$ and sum of phytosterol (**) levels were higher for HS, CF-PS subjects and CF-PI patients supplemented with a higher dose of pancreatic enzymes than in the group CF-PI supplemented with a lower dose $(p<0.0001)$.

\section{Discussion}

This is the first study to assess lathosterol/campesterol and lathosterol/ $\beta$-sitosterol ratios in CF with a concurrent analysis of sterols intake and dose of pancreatic enzymes. Higher concentrations of lathosterol and lower campesterol and $\beta$-sitosterol levels were noted in CF patients, indicating that the synthesis and absorption ratios were higher in CF patients than in HS. The daily intake of energy, fat and cholesterol was higher in CF patients than in HS; however, the daily intake of phytosterols was comparable. Serum total cholesterol and its fractions were lower in CF than in HS, which was related to phytosterol concentrations, age, dose of pancreatic enzymes, nutritional status and genotype. Furthermore, sitosterol and campesterol concentrations were predicted by genotype and pancreatic insufficiency, suggesting that the serum sterol profile in pancreaticinsufficient CF patients is strongly associated with the dose of pancreatic enzymes.

Hypocholesterolemia has been reported in CF, as evidenced by our previous analysis [7] and studies from other centres [32,33]. The reduction in total cholesterol and its fractions occurs in CF children, especially those with pancreatic insufficiency [34]. In our study, only seven (12.8\%) CF patients were pancreatic-sufficient, so it is difficult to speculate about the impact of pancreatic sufficiency on the lipid profile. However, LDL-C concentrations were higher in pancreatic-sufficient than pancreatic-insufficient $C F$ patients (Table 4). The multiple regression analysis identified the severe CFTR genotype as a predictor of lower total and LDL cholesterol concentrations. A study in a CF animal model showed that the exocytosis of cholesterol is impaired [10]. CFTR is a cAMP-activated chloride channel gated by the ATP, which structurally resembles the ATP-binding cassette $(\mathrm{ABC})$. The ABCA1 protein controls the transport of cholesterol and phospholipids, presumably removing sterols from cells $[35,36]$. CFTR mutations trigger the cAMP pathway, 
leading to the inactivation of proteins involved in sterol exocytosis and, consequently, to the intracellular accumulation of cholesterol $[37,38]$.

Our results show abnormalities in sterol homeostasis markers. Lathosterol is the most reliable surrogate marker of de novo liver cholesterol synthesis [39], which can be produced in other tissues but does not pass into the bloodstream; hence, it is considered to be liver-specific [40,41]. Higher lathosterol concentrations may be directly related to CFTR mutations. The in vitro data showed that a CFTR mutation might influence cholesterol trafficking to the plasma membrane and is associated with an increased expression of the cholesterol transport protein NPC1 in CF cells [42]. Fang et al. also reported that CFTR influences cholesterol trafficking to the plasma membrane, which, when depleted, leads to an increase in de novo cholesterol synthesis to restore the membrane content [9].

Campesterol and sitosterol were measured in our study as surrogate markers of sterol absorption [43] and their increased malabsorption was confirmed. We documented low phytosterol concentrations in CF patients with a severe CFTR genotype and exocrine pancreatic insufficiency. The association between low sterols and severe CFTR is in agreement with the very high grade of pancreatic insufficiency in CF patients with the F508del homozygous genotype [44]. Gelzo et al. suggested that pancreatic insufficiency cannot be considered as the only and sufficient explanation of decreased phytosterol levels in CF $[18,19]$; however, they did not perform additional analysis to confirm their suggestion. Reduced absorption of sterols could be caused by liver disease and the impaired secretion of biliary salts, which are essential cofactors of cholesterol esterase that helps to absorb cholesterol [45]. However, our study did not confirm the effect of liver disease on the observed lipid disturbances, indicating the association with the dose of pancreatic enzymes. Further, other factors should be considered, such as the lower (negligible) cholesterol esterase activity of commercially available (pig) enzymes [42], intestinal mucosal abnormalities, lower alkaline duodenal $\mathrm{pH}$, changed bile salt profile or small intestinal bacterial overgrowth, which are present in a significant percentage (35-37\%) of CF patients [45-49]. Interestingly, in the present study, multiple regression analyses showed that campesterol and $\beta$-sitosterol levels were predictors of high cholesterol and its fractions in patients with CF. However, CF dyslipidemia comprises hypocholesterolemia, predominantly in PI patients [7]. Serum sterol concentrations at diagnosis in a CF neonatal screening programme could be an interesting perspective for future studies to define pancreatic status [50].

Standard CF therapy includes a high-energy, high-fat diet (with pancreatic enzyme replacement therapy) [13,51], and it is recommended to use good-quality fats comprising polyunsaturated fatty acids from oils, nuts and avocados (products that are also a source of plant sterols) $[13,30,52]$. Regardless of the guidelines, the phytosterol intake in the present study was comparable in CF patients and HS as a result of the high consumption of saturated fatty acids, butter, eggs and dairy, which was also documented by our previous research [53]. Without any doubt, nutritional recommendations demand individualization. Some patients may have the same energy requirements as healthy peers with no need for a high fat intake. The particular emphasis should be put on the qualitative aspect of the diet comprising, among others, the determination of the fatty acid profile $[13,54,55]$.

The main limitation of this study is its cross-sectional character, which makes inferring causality impossible. Moreover, the reliability of food diaries always, even in highly educated CF patients, raises some doubts; however, we aimed to maximise the precision of the nutritional assessment through education and communication with the study participants. The estimation of the phytosterol content of foods is also challenging, so we created a database from various information sources.

\section{Conclusions}

Endogenous liver production of cholesterol is increased, whereas intestinal sterol absorption is decreased in CF patients. Independent determinants of hypocholesterolemia and low levels of campesterol and $\beta$-sitosterol suggest that malabsorption may play a significant role in sterol abnormalities. A better serum sterol profile in pancreatic-insufficient 
patients is associated with high doses of pancreatic enzymes. Therefore, the measurement of campesterol and $\beta$-sitosterol concentrations in CF patients may potentially serve for the assessment of the effectiveness of pancreatic enzyme replacement therapy and/or compliance with the given recommendations, but further research is required.

Author Contributions: S.D.-C. and J.W. conceived the study, S.D.-C., J.G.-S., A.L. and E.M. recruited patients; S.D.-C., A.L., V.K. and D.W. performed analyses; S.D.-C. and J.W. drafted the manuscript; P.K.-J., K.D. and S.K. performed laboratory analyses; all authors acquired data and interpreted them, revised the manuscript and approved its final version; S.D.-C. obtained funding; S.D.-C. and J.W. supervised the study. All authors have read and agreed to the published version of the manuscript.

Funding: The study was supported by the Polish National Science Centre (SDC-grant No. 2018/02/ X/NZ5/02592).

Institutional Review Board Statement: The study was approved by the Bioethical Committee of the Poznan University of Medical Sciences, Poznań, Poland (decision no 1225/16). The project was conducted in accordance with the Declaration of Helsinki.

Informed Consent Statement: Informed consent was obtained from all subjects involved in the study.

Data Availability Statement: Data shared in this manuscript are in accordance with consent provided by participants on the use of confidential data. The data presented in this study are available within this article.

Acknowledgments: The authors would like to thank the patients and volunteers for their participation in the study.

Conflicts of Interest: There is no conflict of interest.

\section{References}

1. Hassanzad, M.; Boloursaz, M.R.; Darougar, S.; Tashayoie Nejad, S.; Mohajerani, S.A.; Baghaie, N.; Hashemitari, S.K.; Velayati, A.A. Long Term Outcome of Cystic Fibrosis Patients with Multisystem Evaluation. Adv. Respir. Med. 2016, 84, 310-315. [CrossRef]

2. Rich, D.P.; Anderson, M.P.; Gregory, R.J.; Cheng, S.H.; Paul, S.; Jefferson, D.M.; McCann, J.D.; Klinger, K.W.; Smith, A.E.; Welsh, M.J. Expression of Cystic Fibrosis Transmembrane Conductance Regulator Corrects Defective Chloride Channel Regulation in Cystic Fibrosis Airway Epithelial Cells. Nature 1990, 347, 358-363. [CrossRef]

3. Walkowiak, J.; Lisowska, A.; Blaszczyński, M. The Changing Face of the Exocrine Pancreas in Cystic Fibrosis: Pancreatic Sufficiency, Pancreatitis and Genotype. Eur. J. Gastroenterol. Hepatol. 2008, 20, 157-160. [CrossRef]

4. Nash, K.L.; Allison, M.E.; McKeon, D.; Lomas, D.J.; Haworth, C.S.; Bilton, D.; Alexander, G.J.M. A Single Centre Experience of Liver Disease in Adults with Cystic Fibrosis 1995-2006. J. Cyst. Fibros. 2008, 7, 252-257. [CrossRef]

5. Zabner, J.; Smith, J.J.; Karp, P.H.; Widdicombe, J.H.; Welsh, M.J. Loss of CFTR Chloride Channels Alters Salt Absorption by Cystic Fibrosis Airway Epithelia in Vitro. Mol. Cell 1998, 2, 397-403. [CrossRef]

6. Figueroa, V.; Milla, C.; Parks, E.J.; Schwarzenberg, S.J.; Moran, A. Abnormal Lipid Concentrations in Cystic Fibrosis. Am. J. Clin. Nutr. 2002, 75, 1005-1011. [CrossRef] [PubMed]

7. Nowak, J.K.; Szczepanik, M.; Wojsyk-Banaszak, I.; Mądry, E.; Wykrętowicz, A.; Krzyżanowska-Jankowska, P.; Drzymała-Czyż, S.; Nowicka, A.; Pogorzelski, A.; Sapiejka, E.; et al. Cystic Fibrosis Dyslipidaemia: A Cross-Sectional Study. J. Cyst. Fibros. 2019, 18, 566-571. [CrossRef] [PubMed]

8. Peretti, N.; Roy, C.C.; Drouin, E.; Seidman, E.; Brochu, P.; Casimir, G.; Levy, E. Abnormal Intracellular Lipid Processing Contributes to Fat Malabsorption in Cystic Fibrosis Patients. Am. J. Physiol. Gastrointest. Liver Physiol. 2006, 290, 609-615. [CrossRef] [PubMed]

9. $\quad$ Fang, D.; West, R.H.; Manson, M.E.; Ruddy, J.; Jiang, D.; Previs, S.F.; Sonawane, N.D.; Burgess, J.D.; Kelley, T.J. Increased Plasma Membrane Cholesterol in Cystic Fibrosis Cells Correlates with CFTR Genotype and Depends on de Novo Cholesterol Synthesis. Respir. Res. 2010, 11, 61. [CrossRef] [PubMed]

10. White, N.M.; Jiang, D.; Burgess, J.D.; Bederman, I.R.; Previs, S.F.; Kelley, T.J. Altered Cholesterol Homeostasis in Cultured and in Vivo Models of Cystic Fibrosis. Am. J. Physiol. Lung Cell. Mol. Physiol. 2007, 292, 476-486. [CrossRef]

11. Diwakar, V.; Pearson, L.; Beath, S. Liver Disease in Children with Cystic Fibrosis. Paediatr. Respir. Rev. 2001, 2, 340-349. [CrossRef]

12. Narkewicz, M.R. Markers of Cystic Fibrosis-Associated Liver Disease. J. Pediatr. Gastroenterol. Nutr. 2001, 32, 421-422. [CrossRef]

13. Turck, D.; Braegger, C.P.; Colombo, C.; Declercq, D.; Morton, A.; Pancheva, R.; Robberecht, E.; Stern, M.; Strandvik, B.; Wolfe, S.; et al. ESPEN-ESPGHAN-ECFS Guidelines on Nutrition Care for Infants, Children, and Adults with Cystic Fibrosis. Clin. Nutr. 2016. [CrossRef]

14. Cabral, C.E.; Klein, M.R.S.T. Phytosterols in the Treatment of Hypercholesterolemia and Prevention of Cardiovascular Diseases. Arq. Bras. Cardiol. 2017, 109, 475-482. [CrossRef] 
15. Jesch, E.D.; Carr, T.P. Food Ingredients That Inhibit Cholesterol Absorption. Prev. Nutr. Food Sci. 2017, $22,67-80$.

16. Dumolt, J.H.; Rideout, T.C. The Lipid-Lowering Effects and Associated Mechanisms of Dietary Phytosterol Supplementation. Curr. Pharm. Des. 2017, 23, 5077-5085. [CrossRef]

17. Wu, A.H.B.; Ruan, W.; Todd, J.; Lynch, K.L. Biological Variation of $\beta$-Sitosterol, Campesterol, and Lathosterol as Cholesterol Absorption and Synthesis Biomarkers. Clin. Chim. Acta Int. J. Clin. Chem. 2014, 430, 43-47. [CrossRef]

18. Gelzo, M.; Sica, C.; Elce, A.; Dello Russo, A.; Iacotucci, P.; Carnovale, V.; Raia, V.; Salvatore, D.; Corso, G.; Castaldo, G. Reduced Absorption and Enhanced Synthesis of Cholesterol in Patients with Cystic Fibrosis: A Preliminary Study of Plasma Sterols. Clin. Chem. Lab. Med. 2016, 54, 1461-1466. [CrossRef] [PubMed]

19. Gelzo, M.; Iacotucci, P.; Sica, C.; Liguori, R.; Comegna, M.; Carnovale, V.; Dello Russo, A.; Corso, G.; Castaldo, G. Influence of Pancreatic Status on Circulating Plasma Sterols in Patients with Cystic Fibrosis. Clin. Chem. Lab. Med. CCLM 2020, 0. [CrossRef] [PubMed]

20. Farrell, P.M.; Rosenstein, B.J.; White, T.B.; Accurso, F.J.; Castellani, C.; Cutting, G.R.; Durie, P.R.; Legrys, V.A.; Massie, J.; Parad R.B.; et al. Guidelines for Diagnosis of Cystic Fibrosis in Newborns through Older Adults: Cystic Fibrosis Foundation Consensus Report. J. Pediatr. 2008, 153, 4-14. [CrossRef]

21. Walkowiak, J. Assessment of Maldigestion in Cystic Fibrosis. J. Pediatr. 2004, 145, 285-287. [CrossRef] [PubMed]

22. Walkowiak, J.; Nousia-Arvanitakis, S.; Cade, A.; Kashirskaya, N.; Piotrowski, R.; Strzykala, K.; Kouniou, M.; Pogorzelski, A.; Sands, D.; Kapranov, N. Fecal Elastase-1 Cut-off Levels in the Assessment of Exocrine Pancreatic Function in Cystic Fibrosis. J. Cyst. Fibros. 2002, 1, 260-264. [CrossRef]

23. Debray, D.; Kelly, D.; Houwen, R.; Strandvik, B.; Colombo, C. Best Practice Guidance for the Diagnosis and Management of Cystic Fibrosis-Associated Liver Disease. J. Cyst. Fibros. 2011, 10, 29-36. [CrossRef]

24. Castellani, C.; Duff, A.J.A.; Bell, S.C.; Heijerman, H.G.M.; Munck, A.; Ratjen, F.; Sermet-Gaudelus, I.; Southern, K.W.; Barben, J.; Flume, P.A.; et al. ECFS Best Practice Guidelines: The 2018 Revision. J. Cyst. Fibros. 2018, 17, 153-178. [CrossRef]

25. Moran, A.; Pillay, K.; Becker, D.J.; Acerini, C.L. Management of Cystic Fibrosis-Related Diabetes in Children and Adolescents: Management of Cystic Fibrosis-Related Diabetes. Pediatr. Diabetes 2014, 15, 65-76. [CrossRef] [PubMed]

26. McKone, E.F.; Emerson, S.S.; Edwards, K.L.; Aitken, M.L. Effect of Genotype on Phenotype and Mortality in Cystic Fibrosis: A Retrospective Cohort Study. Lancet Lond. Engl. 2003, 361, 1671-1676. [CrossRef]

27. De Boeck, K.; Amaral, M.D. Progress in Therapies for Cystic Fibrosis. Lancet Respir. Med. 2016, 4, 662-674. [CrossRef]

28. Corso, G.; Gelzo, M.; Barone, R.; Clericuzio, S.; Pianese, P.; Nappi, A.; Dello Russo, A. Sterol Profiles in Plasma and Erythrocyte Membranes in Patients with Smith-Lemli-Opitz Syndrome: A Six-Year Experience. Clin. Chem. Lab. Med. 2011, 49, 2039-2046. [CrossRef]

29. Andersson, S.W.; Skinner, J.; Ellegård, L.; Welch, A.A.; Bingham, S.; Mulligan, A.; Andersson, H.; Khaw, K.-T. Intake of Dietary Plant Sterols Is Inversely Related to Serum Cholesterol Concentration in Men and Women in the EPIC Norfolk Population: A Cross-Sectional Study. Eur. J. Clin. Nutr. 2004, 58, 1378-1385. [CrossRef]

30. Normén, A.L.; Brants, H.A.; Voorrips, L.E.; Andersson, H.A.; van den Brandt, P.A.; Goldbohm, R.A. Plant Sterol Intakes and Colorectal Cancer Risk in the Netherlands Cohort Study on Diet and Cancer. Am. J. Clin. Nutr. 2001, 74, 141-148. [CrossRef]

31. Georgiopoulou, V.V.; Denker, A.; Bishop, K.L.; Brown, J.M.; Hirsh, B.; Wolfenden, L.; Sperling, L. Metabolic Abnormalities in Adults with Cystic Fibrosis. Respirol. Carlton Vic 2010, 15, 823-829. [CrossRef]

32. Ishimo, M.-C.; Belson, L.; Ziai, S.; Levy, E.; Berthiaume, Y.; Coderre, L.; Rabasa-Lhoret, R. Hypertriglyceridemia Is Associated with Insulin Levels in Adult Cystic Fibrosis Patients. J. Cyst. Fibros. 2013, 12, 271-276. [CrossRef]

33. González Jiménez, D.; Bousoño García, C.; Rivas Crespo, M.F.; Díaz Martín, J.J.; Acuña Quirós, M.D.; Heredia González, S.; Sojo Aguirre, A.; Garagorri Otero, J.M. [Insulin resistance in overweight cystic fibrosis paediatric patients]. An. Pediatr. Barc. Spain 2003 2012, 76, 279-284.

34. Van der Deen, M.; de Vries, E.G.E.; Timens, W.; Scheper, R.J.; Timmer-Bosscha, H.; Postma, D.S. ATP-Binding Cassette (ABC) Transporters in Normal and Pathological Lung. Respir. Res. 2005, 6, 59. [CrossRef] [PubMed]

35. Brooks-Wilson, A.; Marcil, M.; Clee, S.M.; Zhang, L.H.; Roomp, K.; van Dam, M.; Yu, L.; Brewer, C.; Collins, J.A.; Molhuizen, H.O.; et al. Mutations in ABC1 in Tangier Disease and Familial High-Density Lipoprotein Deficiency. Nat. Genet. 1999, 22, 336-345. [CrossRef] [PubMed]

36. Manson, M.E.; Corey, D.A.; White, N.M.; Kelley, T.J. CAMP-Mediated Regulation of Cholesterol Accumulation in Cystic Fibrosis and Niemann-Pick Type C Cells. Am. J. Physiol. Lung Cell. Mol. Physiol. 2008, 295, 809-819. [CrossRef]

37. Cianciola, N.L.; Carlin, C.R.; Kelley, T.J. Molecular Pathways for Intracellular Cholesterol Accumulation: Common Pathogenic Mechanisms in Niemann-Pick Disease Type C and Cystic Fibrosis. Arch. Biochem. Biophys. 2011, 515, 54-63. [CrossRef] [PubMed]

38. Nissinen, M.J.; Gylling, H.; Miettinen, T.A. Responses of Surrogate Markers of Cholesterol Absorption and Synthesis to Changes in Cholesterol Metabolism during Various Amounts of Fat and Cholesterol Feeding among Healthy Men. Br. J. Nutr. 2008, 99, 370-378. [CrossRef]

39. Stellaard, F.; Lütjohann, D. The Interpretation of Cholesterol Balance Derived Synthesis Data and Surrogate Noncholesterol Plasma Markers for Cholesterol Synthesis under Lipid Lowering Therapies. Cholesterol 2017, 2017, 5046294. [CrossRef]

40. Björkhem, I.; Miettinen, T.; Reihnér, E.; Ewerth, S.; Angelin, B.; Einarsson, K. Correlation between Serum Levels of Some Cholesterol Precursors and Activity of HMG-CoA Reductase in Human Liver. J. Lipid Res. 1987, 28, 1137-1143. [CrossRef] 
41. White, N.M.; Corey, D.A.; Kelley, T.J. Mechanistic Similarities between Cultured Cell Models of Cystic Fibrosis and Niemann-Pick Type C. Am. J. Respir. Cell Mol. Biol. 2004, 31, 538-543. [CrossRef] [PubMed]

42. Andrade, I.; Santos, L.; Ramos, F. Cholesterol Absorption and Synthesis Markers in Portuguese Hypercholesterolemic Adults: A Cross-Sectional Study. Eur. J. Intern. Med. 2016, 28, 85-90. [CrossRef] [PubMed]

43. Walkowiak, J.; Herzig, K.H.; Witt, M.; Pogorzelski, A.; Piotrowski, R.; Barra, E.; Sobczynska-Tomaszewska, A.; TrawinskaBartnicka, M.; Strzykala, K.; Cichy, W.; et al. Analysis of Exocrine Pancreatic Function in Cystic Fibrosis: One Mild CFTR Mutation Does Not Exclude Pancreatic Insufficiency. Eur. J. Clin. Invest. 2001, 31, 796-801. [CrossRef]

44. Peretti, N.; Marcil, V.; Drouin, E.; Levy, E. Mechanisms of Lipid Malabsorption in Cystic Fibrosis: The Impact of Essential Fatty Acids Deficiency. Nutr. Metab. 2005, 2, 11. [CrossRef]

45. Wouthuyzen-Bakker, M.; Bodewes, F.A.; Verkade, H.J. Persistent Fat Malabsorption in Cystic Fibrosis; Lessons from Patients and Mice. J. Cyst. Fibros. 2011, 10, 150-158. [CrossRef] [PubMed]

46. Lisowska, A.; Madry, E.; Pogorzelski, A.; Szydłowski, J.; Radzikowski, A.; Walkowiak, J. Small Intestine Bacterial Overgrowth Does Not Correspond to Intestinal Inflammation in Cystic Fibrosis. Scand. J. Clin. Lab. Invest. 2010, 70, 322-326. [CrossRef]

47. Lisowska, A.; Pogorzelski, A.; Oracz, G.; Siuda, K.; Skorupa, W.; Rachel, M.; Cofta, S.; Piorunek, T.; Walkowiak, J. Oral Antibiotic Therapy Improves Fat Absorption in Cystic Fibrosis Patients with Small Intestine Bacterial Overgrowth. J. Cyst. Fibros. 2011, 10, 418-421. [CrossRef]

48. Ogobuiro, I.; Tuma, F. Physiology, Gastrointestinal; StatPearls Publishing: Treasure Island, FL, USA, 2019.

49. Terlizzi, V.; Tosco, A.; Tomaiuolo, R.; Sepe, A.; Amato, N.; Casale, A.; Mercogliano, C.; De Gregorio, F.; Improta, F.; Elce, A.; et al. Prediction of Acute Pancreatitis Risk Based on PIP Score in Children with Cystic Fibrosis. J. Cyst. Fibros. 2014, 13, 579-584. [CrossRef]

50. Gaskin, K.J. Nutritional Care in Children with Cystic Fibrosis: Are Our Patients Becoming Better? Eur. J. Clin. Nutr. 2013, 67, 558-564. [CrossRef]

51. Strandvik, B.; O Neal, W.K.; Ali, M.A.; Hammar, U. Low Linoleic and High Docosahexaenoic Acids in a Severe Phenotype of Transgenic Cystic Fibrosis Mice. Exp. Biol. Med. Maywood NJ 2018, 243, 496-503. [CrossRef] [PubMed]

52. Drzymała-Czyż, S.; Krzyżanowska, P.; Koletzko, B.; Nowak, J.; Miśkiewicz-Chotnicka, A.; Moczko, J.; Lisowska, A.; Walkowiak, J. Determinants of Serum Glycerophospholipid Fatty Acids in Cystic Fibrosis. Int. J. Mol. Sci. 2017, 18, 185. [CrossRef] [PubMed]

53. Walkowiak, J.; Wilczynski, M.; Boleslawska, I.; Krawczynski, M.; Korzon, M.; Przyslawski, J. The Predominance of Omega6 Polyunsaturated Fatty Acids in Cystic Fibrosis despite Low Arachidonic Acid Levels. Acta Paediatr. 2003, 92, $1354-1355$. [CrossRef] [PubMed]

54. Walkowiak, J.; Lisowska, A.; Blaszczynski, M.; Przyslawski, J.; Walczak, M. Polyunsaturated Fatty Acids in Cystic Fibrosis Are Related to Nutrition and Clinical Expression of the Disease. J. Pediatr. Gastroenterol. Nutr. 2007, 45, 488-489. [CrossRef] [PubMed]

55. van der Haak, N.; King, S.J.; Crowder, T.; Kench, A.; Painter, C.; Saxby, N. Nutrition Guidelines for Cystic Fibrosis in Australia and New Zealand Authorship Group and Interdisciplinary Steering Committee Highlights from the Nutrition Guidelines for Cystic Fibrosis in Australia and New Zealand. J. Cyst. Fibros. 2020, 19, 16-25. [CrossRef] 